\title{
Gletscherschrammen bei Burgstemmen südlich von Hannover
}

\author{
Von Heinz Jordan und Klaus-Dieter Meyer, Hannover
}

Mit 2 Tabellen und 2 Abbildungen

$\mathrm{Z}$ us a mmenfassung. Ein neues Vorkommen von Gletscherschrammen auf Oberem Muschelkalk bei Burgstemmen, $30 \mathrm{~km}$ südlich Hannover, wird beschrieben. Von den sich kreuzenden 2 Schrammensystemen $-\mathrm{N} 10^{\circ} \mathrm{E}$ und $\mathrm{N} 60^{\circ} \mathrm{E}-$ ist das letztere das jüngere. $\mathrm{Da}$ die Schrammen von drenthezeitlichen Kiesen überlagert werden, ist vorerst nicht zu entscheiden, ob die Schrammen durch das Elster-Eis oder durch einen älteren Drenthe-Vorstoß entstanden sind.

A bstract. In this paper a description is given of a new occurrence of glacial striae on Upper Muschelkalk near Burgstemmen, $30 \mathrm{~km}$ south of Hannover. Of the two crossing striation systems $-\mathrm{N} 10^{\circ} \mathrm{E}$ and $\mathrm{N} 60^{\circ} \mathrm{E}-$ the latter is the younger one. Since the striae are overlain by gravels of the Drenthe stadium it is not possible up to now to decide whether the striae have been caused by the Elster ice or by an older glacial advance of the Drenthe stadium.

An den nördlichen Ausläufern der Mittelgebirge, die das Norddeutsche Flachland im Süden begrenzen, ist seit dem vorigen Jahrhundert eine Reihe von Aufschlüssen beschrieben worden, in denen die Oberfläche des anstehenden mesozoischen oder paläozoischen Gesteins glazial geschrammt war. Als erster hatte A. v. Morlot 1844 die Schliffflächen der Hohburger Porphyre bei Wurzen östlich von Leipzig als Beweis angeführt, daß das skandinavische Inlandeis im Pleistozän bis nach Sachsen vorgedrungen sei (vgl. NaumanN 1961 und Wagenbreth 1961).

In Sachsen und Sachsen-Anhalt wurden seit dem historischen Vortrag Torell's (1875) rund 25 Fundpunkte von Glazialschrammen in der Literatur bekanntgemacht (vgl. Grahmann 1957, S. 230 und Schulz 1962, S. 47). Weiter nordwestlich sind die Funde seltener und, wie Tab. 1 und Abb. 1 zeigen, im wesentlichen auf den Flechtinger Höhenzug beschränkt.

$\mathrm{Zu}$ diesen Fundpunkten kommt nun der neuentdeckte bei Burgstemmen hinzu.

Im September 1966 wurde bei B u r g s t e m m e n, 2 km nordöstlich von Elze a. d. Leine, in einer Kiesgrube der Kies- und Mörtelwerke Nord (Firma E. Franke) unter ca. $15 \mathrm{~m}$ mächtigen pleistozänen Sedimenten an der Sohle anstehender Kalkstein angetroffen.

Ta belle 1

Verzeichnis der Gletscherschrammen-Fundpunkte vom Flechtinger Höhenzug bis Osnabrück. Alle Richtungen sind von $\mathrm{N}$ über E gemessen. ält. = älteres, jüng. = jüngeres Schrammensystem mit der vom jeweiligen Autor angegebenen Eisbewegungsrichtung.

\begin{tabular}{|c|c|c|}
\hline Lokalität (Literatur) & geschrammtes Gestein & $\begin{array}{l}\text { Richtung der Schrammen } \\
\text { bzw. der Eisbewegung }\end{array}$ \\
\hline Piesberg bei Osnabrück (НАмм 1882) & Oberkarbon-Sandstein & 10 bis $15^{\circ}$ (nach $\left.S\right)$ \\
\hline Handelah bei Eime (Menzel 1903) & Trochitenkalk & nach SSE \\
\hline $\begin{array}{l}\text { Velpke-Danndorf (WAHNSCHAFFE 1880, } \\
1883 \text { b, 1899; K. RichtER 1962) }\end{array}$ & Rhät-Sandstein & $\begin{array}{l}\text { ält. : } 27^{\circ} \text { (nach SSW) } \\
\text { jüng.: } 84^{\circ} \text { (nach E) }\end{array}$ \\
\hline Groß-Wanzleben (SchüTZE 1900) & Ob. Wellenkalk & fast genau $\mathrm{E}-\mathrm{W}$ \\
\hline Flechtingen (WIEGERS 1907) & Kulm-Grauwacke & 101 und $80^{\circ}$ (gleichzeitig) \\
\hline Hundisburg (WAHNSCHAFFE 1899) & Kulm-Grauwacke & $\begin{array}{l}\text { ält. : } 68^{\circ} \text { (nach SSW) } \\
\text { jüng.: } 43^{\circ} \text { (nach SW) }\end{array}$ \\
\hline Magdeburg (SCHREIBER 1889) & Kulm-Grauwacke & $84^{\circ}$ (nach E) \\
\hline Gommern (WAHNSCHAFFE 1883 a) & Unterkarbon-Sandstein & $\begin{array}{l}\text { ält. : } \quad 6^{\circ} \text { (nach S) } \\
\text { jüng.: } 155^{\circ} \text { (nach SSE) }\end{array}$ \\
\hline
\end{tabular}




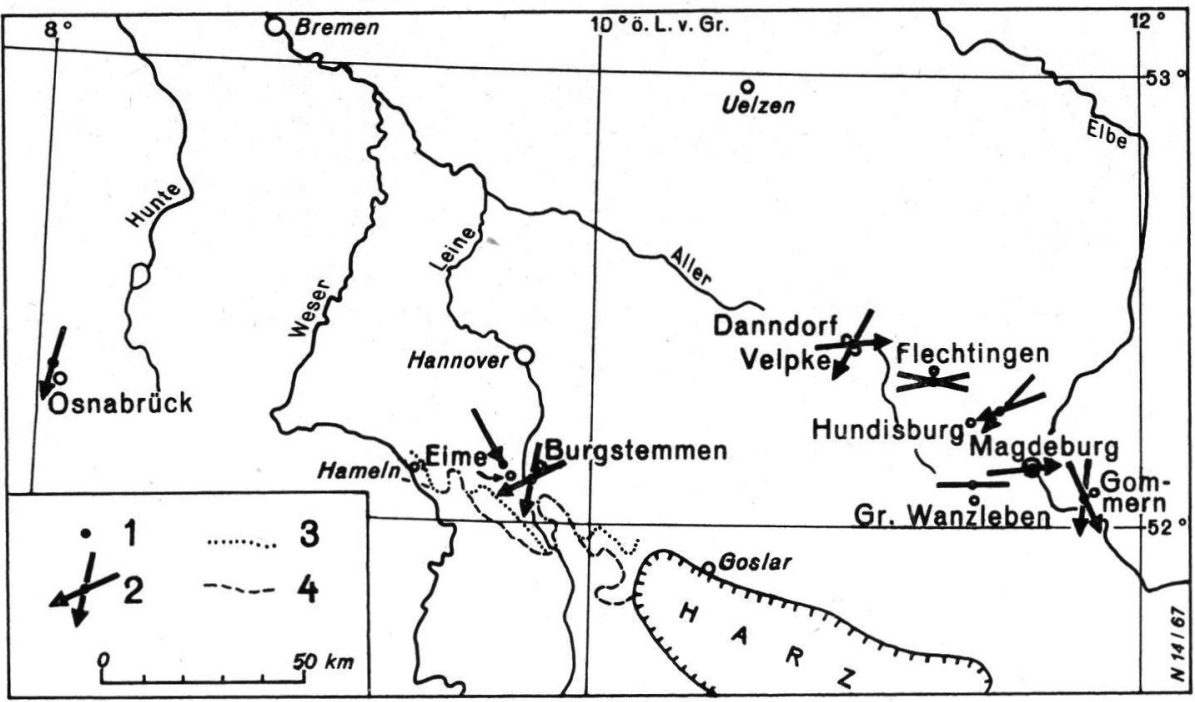

Abb. 1. Verbreitung der Gletscherschrammen vom Flechtinger Höhenzug bis Osnabrück. $1=$ Fundpunkt der Gletscherschrammen; 2 = Richtung der Gletscherschrammen (älteres System unterbrochen); 3 =Südrand des Saale-Eises; 4 = Südrand des Elster-Eises (3 u. 4 nach LütTig 1954).

Der Fundpunkt liegt auf Blatt Elze der Top. Karte 1:25000, bei R: 52 830, H: 78420 und bei ca. $77 \mathrm{~m}$ über NN. Da der Kalkstein von zwei sich senkrecht schneidenden, bankrechten Kluftscharen zertrennt wird, erinnerte er an Mauerwerk, weshalb der Grubenbesitzer, Herr Bode, den Direktor des Alfelder Heimatmuseums, Herrn W. Barner, benachrichtigte, der das Vorkommen besichtigte, sauber freilegen ließ und seinerseits dem Niedersächsischen Landesamt für Bodenforschung Mitteilung machte.

Dank dieser freundlichen Mitteilung und dank dem Entgegenkommen von Herrn BODE konnte der erstgenannte der Verf. am 13. 9. 1966 die Kiesgrube mit dem KalksteinVorkommen besuchen. Freigelegt war auf ca. $5 \mathrm{~m}$ mal $6 \mathrm{~m}$ die Schichtfläche einer flachliegenden, 1 bis $2 \mathrm{dm}$ mächtigen Kalksteinbank der "Tonplatten“ (Oberer Muschelkalk). Ein Schurf an ihrer Nordseite ergab, daß die Schicht mitsamt den darunter folgenden hier über $1 / 2 \mathrm{~m}$ tief erodiert war. Wenige Meter westlich und etwas höher lag eine zweite, isolierte, flach nach $\mathrm{E}$ einfallende Kalkstein-Bank von ca. $2 \mathrm{~m}$ mal $2 \mathrm{~m}$ Fläche frei, die an einer Ecke glazial geschrammt war.

Es waren zwei Schliffrichtungen zu erkennen (Abb. 2): Ein System von relativ breiten und tiefen Schrammen, die streckenweise wieder abgeschliffen waren, streicht mit $10^{\circ}$ etwa N-S. Eine zweite, mit $60^{\circ}$ WSW-gerichtete Schar von feineren, aber viel besser erhaltenen Kritzen kreuzt und kappt z. T. die N-S-Schrammen, ist also jünger als jene.

Einige der Schrammen beginnen mit einer relativ breiten Vertiefung, die eindeutig das Einsetzen des kritzenden Geschiebes bezeichnet. Es sei darauf hingewiesen, daß WAHNSCHAFFE (1880, S. 793; 1899, S. 57, 59) bei Velpke und Hundisburg „keilförmige Figuren“ beschreibt, „... deren Spitze das Einsetzen des Geschiebes bedeutet, während der sich verbreiternde Keil die Bewegungsrichtung des Gletschers anzeigt". - Diese Figuren sind also entgegengesetzt orientiert zu denen bei Burgstemmen.

Die Kalksteinbank wird unmittelbar überlagert von Mittel- bis Grobkies mit einzelnen Steinen, der weiter oben dm- bis m-mächtige Einschaltungen von schwach feinkiesigem Sand bis zu reinem Feinsand enthält. Der Kies(-sand) ist stellenweise kreuzgeschichtet und glazifluviatiler Entstehung. Seine Mächtigkeit beträgt über dem Gletscherschliff $13 \mathrm{~m}$, die Lagerung ist an einigen Stellen durch Verwerfungen, wohl durch Toteis 


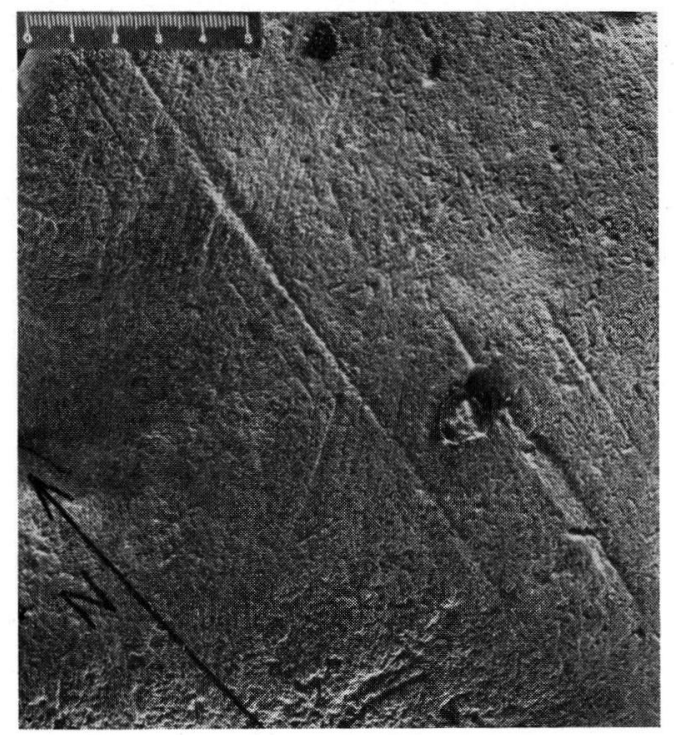

Abb. 2. Zwei Systeme von Gletscherschrammen auf einer Kalksteinbank aus den "Tonplatten“ (Ob. Muschelkalk) ca. $1 \mathrm{~km}$ SSW Burgstemmen. Maßstab $5 \mathrm{~cm}$. Das Belegstück wird im Niedersächsischen Landesamt für Bodenforschung, Hannover-Buchholz, Alfred-Bentz-Haus, aufbewahrt.

verursacht, gestört. Die obersten $2 \mathrm{~m}$ sind, ohne scharfe Untergrenze, durch Eisenlösungen gelbbraun verfärbt. Darüber folgt ein ca. $2 \mathrm{~m}$ mächtiger Geschiebelehm, der von LüTTIG bei der Neuaufnahme des Blattes Elze als saalezeitlich kartiert wurde und auf den Kuppen z. T. erodiert ist, und schließlich ca. ${ }^{1 / 2} \mathrm{~m}$ weichseleiszeitlicher Lößlehm.

Tabelle 2

Vergleich des Drenthe-Kieses der Kiesgrube Franke, Burgstemmen, mit 3 Kiesen vom Leinesteilufer bei Betheln (2 km südlich Burgstemmen) nach LüTTig 1960, S. 375. $\mathrm{N}=$ nordisches, $\mathrm{M}=$ mesozoisch-einheimisches, $\mathrm{P}=$ paläozoisch-einheimisches Material.

\begin{tabular}{|c|c|c|c|c|c|c|}
\hline & \multirow{2}{*}{\multicolumn{3}{|c|}{$\begin{array}{c}\text { B u rgstem m e n } \\
\text { Drenthe-Kies }\end{array}$}} & \multicolumn{3}{|c|}{ Betheln } \\
\hline & & & & \multicolumn{2}{|c|}{ Drenthe-Kies } & Elster-K. \\
\hline & Basis & $3 \mathrm{~m}$ üb. B. & $8 \mathrm{~m}$ üb. B. & Schurf III & Kiesgr. & Wunram \\
\hline & 3535 Ger. & 583 Ger. & 468 Ger. & Probe be & Probe bt & Probe bx \\
\hline $\begin{array}{l}\text { N: Flint } \\
\quad \text { Kristallin } \\
\text { Klast. Sedimente } \\
\text { Kalke }\end{array}$ & $\begin{array}{l}2,7 \\
4,0 \\
2,4 \\
0,4\end{array}$ & $\begin{array}{l}3,3 \\
4,5 \\
0,5 \\
0,5\end{array}$ & $\begin{array}{l}2,8 \\
1,5 \\
1,1 \\
0,2\end{array}$ & $\begin{array}{l}7 \\
1 \\
1\end{array}$ & $\begin{array}{l}1 \\
4 \\
4\end{array}$ & $\begin{array}{l}2 \\
6 \\
7\end{array}$ \\
\hline $\begin{array}{l}\text { M: Buntsandstein } \\
\text { Muschelkalk } \\
\text { Oberkreide-Kalke } \\
\text { Malm-Kalke } \\
\text { Keuper } \\
\text { restl. Mesozoikum } \\
\text { Basalte }\end{array}$ & $\begin{array}{r}35,7 \\
32,2 \\
0,7 \\
0,2 \\
5,3 \\
0,1\end{array}$ & $\begin{array}{r}28,3 \\
29,8 \\
6,9 \\
1,2 \\
5,0 \\
-\end{array}$ & $\begin{array}{r}32,0 \\
30,3 \\
7,9 \\
1,1 \\
5,8 \\
0,2\end{array}$ & $\begin{array}{r}21 \\
9 \\
16 \\
39 \\
1\end{array}$ & $\begin{array}{r}35 \\
22 \\
3 \\
2 \\
18 \\
3\end{array}$ & $\begin{array}{r}31 \\
17 \\
2 \\
3 \\
11 \\
2\end{array}$ \\
\hline $\begin{array}{l}\text { P: TW-Porphyr } \\
\text { Kieselschf. (meist Lydite) } \\
\text { Grauwacke } \\
\text { restl. paläoz. Sedimente }\end{array}$ & $\begin{array}{l}4,7 \\
3,6 \\
0,8 \\
2,6\end{array}$ & $\begin{array}{r}6,5 \\
3,1 \\
7,4\end{array}$ & $\begin{array}{r}3,8 \\
3,2 \\
6,0\end{array}$ & $\begin{array}{r}- \\
1\end{array}$ & $\begin{array}{l}1 \\
1 \\
3 \\
1\end{array}$ & $\begin{array}{l}2 \\
3 \\
3 \\
5\end{array}$ \\
\hline Quarz & 4,6 & 3,1 & 4,1 & 3 & 2 & 6 \\
\hline $\mathrm{N}: \mathrm{M}$ (einschl. Quarz) : P & $10: 78: 12$ & $9: 74: 17$ & $6: 81: 13$ & $9: 89: 2$ & $9: 85: 6$ & $15: 72: 13$ \\
\hline
\end{tabular}


An einer dem Gletscherschliff benachbarten Stelle wurde unmittelbar über dem Kalkstein die unterste, $1 / 2 \mathrm{~m}$ mächtige Kieslage geschiebekundlich untersucht; $3 \mathrm{~m}$ bzw. $8 \mathrm{~m}$ über der Kiesbasis wurden Kontrollzählungen durchgeführt (s. Tab. 2). Demnach ist der Kies, was die Zusammensetzung der ausgezählten Geröllfraktion von ca. 1 bis $6 \mathrm{~cm}$ Durchmesser betrifft, einigermaßen homogen; nur der Kreidekalk-Anteil nimmt vom Liegenden zum Hangenden deutlich zu. Hauptkomponenten sind der im Untergrund bzw. in naher Umgebung anstehende Muschelkalk und Buntsandstein.

Vergleicht man diesen Kies mit den zahlreichen, von LüTTIG (1960) untersuchten Kiesvorkommen der Umgebung, so ergibt sich folgendes: Ein fluviatiler (Terrassen-) Kies scheidet auch nach der Zusammensetzung aus. Der relativ geringe Anteil an nordischem Sediment und Kristallin deutet eher auf Drenthe- als auf Elsterkies hin. Auffällig ist der für einen Drenthe-Kies sehr hohe Anteil einheimisch-paläozoischen Materials. Offenbar hat der Kies eine Menge Oberterrassen-Material aufgenommen, möglicherweise aus der Gegend von Rössing, 5 km nordöstlich von Burgstemmen, wo nach LüTTIG (1960) Oberterrassen-Kiese verbreitet sind.

Nur so läßt sich der für einen drenthezeitlichen glazifluviatilen Kies zu hohe Anteil an einheimischem Paläozoikum und insbesondere an Thüringer-Wald-Porphyren erklären. Rechnet man von den gezählten 4-6,5\% Porphyren etwa $4 \%$ der aufgearbeiteten Oberterrasse zu, die bei Rössing nach Lütrig (1960, S. 383) 14 bzw. 16\% T-W-Porphyre enthält, so müßte etwa ein Viertel des Burgstemmener Kieses aufgearbeitetes Oberterrassen-Material sein.

An dem nordischen Material des basalen Kieses bestimmte der letztgenannte der Verf. das "Theoretische Geschiebezentrum“ (TGZ, nach Methode LüTTIG) zu 15,36 - 57,60, womit drenthestadiales Alter als bewiesen gelten muß (vgl. LütTIg 1959, S. 387, Abb.12).

Der glazial geschrammte Muschelkalk wird also unmittelbar von glazifluviatilem Kies des Drenthe-Stadiums, dieser von Drenthe-Geschiebelehm überlagert. Die Grundmoräne, deren Geschiebe den festen Untergrund gekritzt haben, ist an den derzeit aufgeschlossenen Stellen erodiert.

Daher läßt sich das Alter der Schrammen z. Zt. nicht genau bestimmen. Man wird sie zuerst einer elstereiszeitlichen Grundmoräne zuschreiben, die von den Schmelzwässern des heranrückenden Drenthe-Eises erodiert wurde. Elster-Geschiebelehm wurde von LüTTIG (1960, S. 378) z.B. 2 km südlich der Kiesgrube, bei Elze, und $2 \mathrm{~km}$ östlich, zwischen Heyersum und Haus Escherde, beschrieben. In den heute auf dem Kalkstein lagernden Vorschüttkiesen sollte man in diesem Fall Material der aufgearbeiteten elstereiszeitlichen Grundmoräne erwarten; dafür gibt jedoch die Geschiebezählung keinen Hinweis, vielmehr liegt das TGZ ausgesprochen tief im drenthestadialen Streubereich.

Man muß also erwägen, daß die Schrammen von einem älteren Vorstoß des DrentheEises herrühren. Nach LüTTIG $(1954,1960)$ hat nämlich das Drenthe-Eis das Leinetal bei Burgstemmen zweimal überfahren, und zwar in der (älteren) Alfelder und in der Fredener Staffel (der Hamelner Phase), die durch den Eisrückzug der Imsener Etappe getrennt sind.

Der hangende Geschiebelehm in der Frankeschen Kiesgrube könnte also in die Fredener Staffel gehören, der glazifluviatile Kies darunter der zugehörige Vorschütt-Kies sein und die für die Schrammen verantwortliche erodierte Grundmoräne in die Alfelder Staffel zu stellen sein.

Theoretisch ist es natürlich auch nicht unmöglich, daß das ältere System elstereiszeitlich, das jüngere drenthezeitlich ist.

In diesem Zusammenhang erhebt sich die Frage, wie groß der Altersunterschied zwischen den älteren N-S- und den jüngeren ENE-WSW gerichteten Schrammen ist. In Velpke und in Gommern erklärte Wahnschaffe (1880, S. 797, bzw. 1883a, S. 837) die 
jüngeren Kritzen, die auch dort von der N-S-Richtung der älteren Schrammen stark abweichen (s.o.), durch eine „Ablenkung des Eisstromes ... vielleicht in einer späteren Periode, als das Gletschereis nicht mehr so mächtig war".

Gegen die Vermutung Penck's, die zwei Schrammensysteme seien durch eine, lokal in wechselnder Richtung bewegte Grundmoräne entstanden, verwahrte sich WAHNSCHAFFE (1883b, S. 226) mit der Begründung, daß sowohl das ältere wie das jüngere System an allen 8 Beobachtungspunkten ihre Richtung streng beibehielten. K. RichtER (1962, S. 128-129) wollte trotzdem das jüngere E-W-System eher durch ,irreguläre Bewegung größerer Blöcke", bedingt durch Gesteinsaufbrüche im Untergrund, erklären als durch eine ins obere Allertal nach Osten abgelenkte jüngere Eiszunge. Im übrigen gibt es sowohl für eine streng lokale (z. B. Demorest 1938) wie für eine regionale Ablenkung des Eisstromes (z. B. LARSSON 1945) zahlreiche gut untersuchte Beispiele.

Im Falle der Burgstemmener Schrammen muß man abwarten, ob bei fortschreitendem Kiesabbau weitere Gletscherschliffe gefunden werden. Sollten sich dann die WSW-gerichteten Schrammen als richtungskonstant erweisen, so können sie nicht mehr als Marken einer ganz lokalen, untergrundsbedingten Zufallsbewegung des Eises angesehen werden. Überdies ist zu hoffen, daß unter dem Kies einmal Reste der Grundmoräne angetroffen werden, deren geschiebekundliche Datierung das Alter der Schrammen klären könnte. Leider ruht gerade an dieser Stelle der Abbau; wie sich bei einem erneuten Besuch der Verfasser am 13. 11. 1966 herausstellte, ist die geschrammte Platte bereits wieder verschüttet. Eine Veröffentlichung sollte unter diesen Umständen nicht länger hinausgeschoben werden, zumal der Gletscherschliff bei Burgstemmen seit nunmehr 60 Jahren der erste neue Schrammenfund in Niedersachsen ist.

\section{Literatur}

Demorest, M.: Ice flowage as revealed by glacial striae. Journ. Geol., 46, 700-725, Chicago 1938. Grahmann, R.: Ausdehnung und Bewegungsrichtung des Inlandeises in Sachsen. Ber. geol. Ges. DDR., 2, 227-232, Berlin 1957.

Hамм: Beobachtungen im Diluvium der Umgegend von Osnabrück. Z. deutsch. geol. Ges., 34, 629-636, Berlin 1882.

Larsson, W.: Zur Kenntnis der spätglazialen Eisbewegungen westlich des Wenersees, Schweden. Sver. geol. Undersökn., Ser. C., 466, 3-13, Stockholm 1945.

LüTtIG, G.: Alt- und mittelpleistozäne Eisrandlagen zwischen Harz und Weser. Geol. Jb., 70, 43-125, Hannover 1954. - - Methodische Fragen der Geschiebeforschung. Geol. Jb., 75, 361-418, Hannover 1958. - - Neue Ergebnisse quartärgeologischer Forschung im Raume Alfeld-Hameln-Elze. Geol. Jb., 77, 337-390, Hannover 1960.

Menzel, H.: Uber Glacialschrammen im südlichen Hannover. Cbl. Miner. Geol. Paläont., 1903, 509-512, Stuttgart 1903.

Naumann, E.: A. v. Morlot zur Inlandeisbedeckung Deutschlands. Geologie, 10, 351-352, Berlin 1961.

Richter, K.: Das Alter der Gletscherschrammen von Velpke. Eiszeitalt. u. Gegenw., 12, 125-130, Ohringen 1962.

Schreiber, A.: Glacialerscheinungen bei Magdeburg. Z. deutsch. geol. Ges., 41, 603-608, Berlin 1889.

Schulz, W.: Gliederung des Pleistozäns in der Umgebung von Halle (Saale). Geologie, Beih. 36, 1-69, Berlin 1962.

SchÜтze, E.: Glacialerscheinungen bei Gross-Wanzleben, unweit Magdeburg. Cbl. Miner. Geol. Paläont., 1900, 85-87, Stuttgart 1900.

Tonell, O.: (Vortrag über Inlandeis in Norddeutschland.) Z. deutsch. geol. Ges., 27, 961-962, Berlin 1875.

Wagenbreth, O.: Aus der Vorgeschichte von Torells Glazialtheorie. Ber. geol. Ges. DDR, 5, 175-190, Berlin 1961. 
WaHnSChafFe, F.: Über Gletschererscheinungen bei Velpke und Danndorf. Z. deutsch. geol. Ges., 32, 774-798, Berlin 1880. - - Uber Glacialerscheinungen bei Gommern unweit Magdeburg. Z. deutsch. geol. Ges., 35, 831-848, Berlin 1883 (1883a). - - Beitrag zur Kenntniss der Rüdersdorfer Glacialerscheinungen. Jb. preuß. geol. L.-A., 1882, 219-227, Berlin 1883 (1883b). - - Uber das Vorkommen von Glacialschrammen auf den Culmbildungen des Magdeburgischen bei Hundisburg. Jb. preuß. geol. L.-A., 19, 52-65, Berlin 1899.

WIEGERs, F.: Uber Glazialschrammen auf der Culmgrauwacke bei Flechtingen. Jb. preuß. geol. L.-A., 25, 472-476, Berlin 1907.

Manuskr. eingeg. 10. 2. 1967.

Anschrift der Verf.: Dipl.-Geologe Dr. Heinz Jordan und Dipl.-Geologe Dr. Klaus-Dieter Meyer, Assessoren des Geol. Dienstes, Niedersächsisches Landesamt für Bodenforschung, 3 Hannover-Buchholz, Alfred-Bentz-Haus. 